Meta

Journal des traducteurs

Translators' Journal

\title{
Traduire, créer
}

\section{Laurence Belingard, Maryvonne Boisseau et Maïca Sanconie}

Volume 62, numéro 3, décembre 2017

La traduction littéraire comme création

URI : https://id.erudit.org/iderudit/1043944ar

DOI : https://doi.org/10.7202/1043944ar

Aller au sommaire du numéro

Éditeur(s)

Les Presses de l’Université de Montréal

ISSN

0026-0452 (imprimé)

1492-1421 (numérique)

Découvrir la revue

Citer ce document

Belingard, L., Boisseau, M. \& Sanconie, M. (2017). Traduire, créer. Meta, 62(3), 489-500. https://doi.org/10.7202/1043944ar d'utilisation que vous pouvez consulter en ligne.

https://apropos.erudit.org/fr/usagers/politique-dutilisation/ 


\title{
PRÉSENTATION \\ Traduire, créer
}

\author{
LAURENCE BELINGARD \\ université d'Avignon, France \\ laurence.belingard@univ-avignon.fr
}

MARYVONNE BOISSEAU

université de Strasbourg, France

maryvonne.boisseau@unistra.fr

MAÏCA SANCONIE

Université d'Avignon, France

marie-francoise.sanconie@univ-avignon.fr

Certes, on traduit toujours à l'ombre du texte source, on traduit toujours après. Et cependant la tâche la plus essentielle du traducteur consiste à faire de cet après le berceau dans lequel le premier texte est secoué par le vent d'une renaissance, où les mots anciens se mettent à sonner comme des mots nouveaux sans que le charme de leur éloignement en soit diminué.

(Antonio Prete 2011/2013: 10)

Issue d'une source textuelle et devenant matière, la traduction traverse le corps du traducteur pour naître dans une autre langue: la traduction est «comme l'acte d'une seconde naissance», nous dit Yasmina Foehr-Janssens (2016: 129). Aussi se pencher sur toute la dimension créatrice de la traduction littéraire implique-t-il un véritable retour au secret de sa naissance.

On a pu nier ce geste créateur en raison de l'autorité du texte à traduire: la traduction, inévitablement, vient après. La plume du traducteur ne saurait rivaliser avec la plume de l'auteur; bien au contraire, elle est à son service. On a pu également nier la dimension créatrice pourtant inhérente au traduire en raison des différentes modalités mêmes de la traduction, depuis l'imitation jusqu'à l'adaptation en passant par la traduction «littérale» ou bien encore la «version» d'après tel ou tel. Cependant, s'il existe bel et bien différents modes de traduction, tous mettent en jeu une relation complexe du traducteur au texte, conditionnée - et contrainte - par un genre de texte, une demande éditoriale particulière, des conditions de traduction spéciales, l'environnement de travail du traducteur ou de la traductrice, et le lectorat visé.

Par ailleurs, il y a traduction et traduction et toute traduction n'est pas création, toute traduction n'est pas œuvre d'art. Il ne viendrait sans doute à l'esprit de personne 
de considérer spontanément une traduction technique comme une œuvre d'art. C'est donc bien la traduction littéraire qui est au premier chef concernée par cette question, et cela d'autant plus que les traducteurs littéraires auxquels on reconnaît officiellement, en France notamment, le statut d'auteur, aspirent à celui d'écrivains à part entière ${ }^{1}$. Elle l'est encore parce que si les sciences cognitives nous enseignent que traductrices et traducteurs sont le plus souvent engagés pour ainsi dire corps et âme dans le processus, il demeure néanmoins difficile d'en saisir le mystère et d'en appréhender les étapes.

Pourtant, et ce volume de Meta le montre, envisager la traduction comme création, comme une création, aller jusqu'à en faire une œuvre d'art, c'est l'aborder sous l'angle de la fertilisation d'une ouvre par une autre, de la continuation d'un rythme dans un autre; c'est en percevoir l'énergie, en reconnaître le savoir-faire, en recevoir la beauté. Il est alors possible de la montrer, défi relevé par la commissaire de l'exposition Après Babel, traduire présentée au MUCEM à Marseille (14 décembre 201620 mars 2017), Barbara Cassin, qui écrit ceci :

La performance nous ramène définitivement à la traduction en tant qu'une traduction est elle-même une œuvre d'art: traduire, au même titre que la peinture ou la poésie, est l'un des beaux-arts. D'abord parce que traduire exige une tekhnê: du savoir, du savoir-faire et du temps, élan et patience, hésitation et décision, culture et intuition, etc. Ensuite parce que, même quand une traduction repose stabilisée comme une œuvre à part entière, une merveille, un achievement, une traduction n'est jamais définitive, mais relève comme l'art d'une énergie continue. Elle est portée par une intention, un désir qui la temporalise - la Bible de Luther, Le Corbeau de Baudelaire, tout comme l'Aristote d'Averroès ou le Freud de Strachey, sont des œuvres d'auteur, datées autant que déterminantes. Avec ce tour de plus, propre à la traduction, qui la rend toujours en contradiction avec elle-même, une mine d'oxymores: chaque traduction est une œuvre qui répond à une œuvre, elle s'efface et elle prend place, elle communique en langue de tous les jours et elle invente un idiome, folle d'audace et de servilité. (Cassin 2016: 250)

Toute la problématique de la traduction littéraire comme création est là, dans cette accumulation de paradoxes. Définie comme «œuvre de l'esprit» dans le code de la Propriété intellectuelle, la traduction se matérialise comme œuvre d'art dans son statut d'inachevé: tout comme le texte dont elle est issue, elle est une proposition, une vision du monde, et par là même coulée dans ce processus de non-finitude qui caractérise l'écriture. Il ne s'agit donc pas de clore une œuvre dans une langue en la traduisant, mais au contraire de l'ouvrir à une autre culture, une autre langue-culture, et par là même au monde. "La langue», dit Prete, "est hospitalière. [...] Traduire, c'est accueillir une autre langue qui se trouve en chemin, à un certain point de son chemin. [...] Traduire, c'est en même temps accueillir avec sa propre langue un livre en chemin et ajouter un nouveau temps, une nouvelle pause au chemin du livre» (Prete 2011/2013: 13,15). Cet accueil est en lui-même propice à la création car il n'y a pas du côté de la langue source un surgissement créateur originel, et du côté de la langue cible, le seul labeur d'un exécutant. Imagine-t-on un musicien se contentant de reproduire les sons d'une partition sans tenter d'en retrouver la musique? Le traducteur - la traductrice - met tous ses sens à la disposition de l'œuvre à traduire. Son écoute, sa sensibilité, autant son corps que son esprit, sont à l'œuvre.

La traduction littéraire comme création nous ramène donc d'abord au traducteur, sujet lisant, traduisant, écrivant. Sa visibilité et sa présence ne devraient venir en 
aucune façon troubler la réception de la traduction, mais au contraire l'éclairer et renseigner sur le rapport à l'autre dont elle témoigne. En effet, le malentendu opposant traduction et création provient, notamment, d'une rivalité fantasmée entre auteur de l'œuvre première et auteur de l'œuvre seconde: le "producteur» de la traduction pourrait ainsi réclamer une part du capital symbolique de l'auteur et empiéter sur ses prérogatives de créateur. Cela suscite deux objections: premièrement, le traducteur, dont le nom n'est pas connu du public, n'en est pas moins créateur et, deuxièmement, le traducteur célèbre - souvent un autre écrivain-traducteur - est au contraire valorisé pour avoir su porter l'œuvre de cet auteur dans sa propre langue. La reconnaissance de cette part de création dans le processus de traduction suffirait probablement à invalider la rivalité auteur/traducteur. Si tel était le cas, la place du traducteur-auteur rejoindrait celle de l'auteur dans le domaine éditorial. Le surgissement d'une œuvre est en réalité un moment - sinon un événement - dans l'histoire de la littérature, dont les conséquences se mesurent à l'aune des créations de nature diverse qu'elle engendre et qui sont autant de signes manifestes de sa dimension universelle. Ses traductions, en diverses langues et selon des modes différents comme les adaptations pour le théâtre ou le cinéma, en sont les multiples facettes.

La traduction littéraire comme création nous ramène aussi au lecteur, à l'auditeur ou au spectateur auquel est destiné le texte traduit ou adapté. La question se pose en effet de la réception des traductions, problème complexe s'il en est. D’un côté, la réception d'une traduction ne semble exister que dans sa dimension éditoriale qui tend à organiser l'effacement du traducteur au profit de la mise en valeur de l'original dans sa version traduite, "version française» ou «italienne», etc.; ce faisant, renvoyant d'ailleurs à la langue plutôt qu'à l'œuvre elle-même dans son intégralité («traduit de...»). D'un autre côté, le lecteur lui-même n'est pas toujours en situation d'apprécier la valeur créative de la traduction s'il ne peut la comparer à l'original, ce qui n'est somme toute que la situation de réception «normale», la traduction - c'est un lieu commun - étant d'abord destinée à un public qui ne connaît pas la langue dans laquelle a été écrite l'œuvre qu'on a traduite. La traduction renvoie donc inévitablement au texte dont elle dérive, confirmé ainsi dans son statut d'original, comme l'écrit Fabienne Durand-Bogaert:

Qu'il surgisse ou non "organiquement», comme le pensait Walter Benjamin, de l'original, le texte d'une traduction est toujours un texte second renvoyant à un texte premier et, de ce fait, le confirmant dans son statut d'«original». (Durand-Bogaert 2014: 12)

De plus, le processus créatif ne se voit pas. Il est tout entier contenu dans la traduction produite et ne se lit, s'entend et s'évalue que s'il laisse les traces d'une inscription reconnaissable du traducteur dans le texte. Ceci pose le problème de la lecture du texte traduit dont l'étude, comme celle de la genèse du processus, n'a pas encore fait l'objet de recherches étendues de la part des traductologues, les mieux à même sans doute de mettre au jour ce qui s'y trame afin de faire la part de ce qui est véritablement créatif et de ce qui ne l'est pas, un peu comme si, dans une perspective critique, on séparait le bon grain de l'ivraie. Encore faut-il avoir accès à cette part intime du travail traductif que constituent les brouillons, les ratures, les recherches des traductrices et traducteurs eux-mêmes, ce que tente d'entreprendre une critique génétique des traductions. Sans cet accès, qui d'ailleurs ne rend compte du processus 
que partiellement, le traductologue en est réduit à émettre des hypothèses fondées sur les types de différences observées entre l'original et la traduction et sur les autres choix qu'il suppose avoir été écartés par le traducteur. La compréhension du processus créatif passe alors aussi par un retour au traducteur, par la compréhension de son rapport aux textes, aux langues, au langage; c'est ainsi s'inquiéter de savoir et connaître "quel être au monde s'est trouvé mis en jeu, dont le texte traduit nous restitue nécessairement quelque chose» (Durand-Bogaert, 2014: 18). De cette façon, le «projet de traduction» (selon l'expression de Berman), le traducteur et la traduction peuvent être pris ensemble dans la réalisation de ce qui pourra être appréhendé comme création et rendu - comme on rend à César ce qui est à César - à son auteurtraducteur.

On comprend donc que la circulation entre les langues dépasse les notions de concessions, compromis, gains et pertes, toutes assujetties aux concepts de fidélité et de trahison d'un original par essence non reproductible. En réalité, elle emprunte des voies sensorielles et cognitives subtiles. Patrick Quillier utilise le terme de «traduction acroamatique», restaurant l'oreille comme fondamentale dans l'entendement et la pratique de la traduction:

Si l'on veut bien se rappeler que «akraoma» désigne la chose entendue au double sens de la perception auditive et de l'intellection, on aura dès lors une perspective nouvelle en matière d'approche théorique et surtout pratique de la traduction littéraire puisqu'aussi bien cette activité est un cas particulier, et tout particulièrement intéressant, de l'usage de l'entendement - que l'on pourra à bon droit nommer démarche «acroamatique». (Quillier 2002: 164)

La création se donne donc à entendre et à voir dans les traductions littéraires, pour qui sait écouter autant que lire, dans ce travail d'échange entre les sens, le sens et les matières des langues: "On peut dire, déclare Cassin, que la traduction est une création littéraire, une création de langue, une création de langue dans une langue» (Cassin 2014: 129). La traduction s'inscrit essentiellement dans une relation, avec l'autre-auteur, avec la matière des langues et avec l'autre-lecteur. Selon Jean-Louis Lebrave, cette relation s'organise notamment au cours du travail lui-même sur ce que la critique génétique appelle les «variantes liées » ou la mise en place d'un «système de variations liées» (Lebrave 2014: 37):

C'est en les mettant au jour et en s'efforçant de les restituer que le processus de traduction lui-même met progressivement en place, d'une manière dont le traducteur n'a pas forcément directement conscience, un système isotopique [au sens d'isotopie textuelle]. Grâce à celui-ci, la compétence du traducteur s'accorde, en un sens très proche du sens musical, au texte à traduire. Cet accord permet au traducteur d'élaborer tout un préconstruit sous-jacent à l'activité de traduction, grâce auquel ses capacités de performance approcheront le texte à traduire d'une manière plus harmonieuse, plus fine, plus riche, plus exhaustive. (Lebrave 2014: 37)

Traduire relèverait alors, in fine, d'une performance, d'une lente et patiente écoute, d'un travail de la matière des mots pour retrouver la forme du texte original dans une souple dimension, l'expulser et la faire re-naître dans ce nouveau contenant qu'est la langue de traduction portant l'empreinte de son autre créateur.

Rendre ses lettres de noblesse à une activité trop souvent considérée comme ancillaire, tel a donc été le projet du colloque «La traduction littéraire comme création» organisé à l'Université d'Avignon les 18 et 19 mai 2015. Ce volume, qui propose 
une sélection des articles écrits par la suite, auxquels s'ajoute une nouvelle contribution, s'en fait l'écho. Les articles rendent compte, d'une part, des tentatives d'approcher au plus près la dimension créatrice de la traduction, à la fois dans le processus traductif lui-même et dans le pouvoir de la traduction à influer sur la création dans son ensemble; et, d'autre part, de mesurer la possible influence, par une sorte d'effet miroir, du texte traduit sur certaines représentations linguistiques et culturelles dans la culture source elle-même. L'histoire de la traduction montre que le traducteur, par les interférences qu'il suscite, entraîne le texte dans un jeu où, au sein même d'un «système» littéraire national, les différents sous-systèmes que représentent notamment les genres littéraires se chevauchent. C'est la théorie du polysystème développée par Itamar Even-Zohar et Gideon Toury (1980-1995). Aussi s'est-il avéré nécessaire de s'approcher au plus près de la créativité au cœur du processus traductif dans toutes ses dimensions.

La première partie du volume examine certains présupposés théoriques relatifs à l'idée de création - et de créativité - à l'œuvre au cours d'un processus qui met en jeu deux ou plusieurs langues à la fois. Cette mise en relief de la difficulté de penser les étapes de la création est réfléchie dans la seconde partie par des études au ras des textes qui visent, à partir du «produit» et des traces inscrites dans le texte, à révéler à la fois l'invisible cheminement cognitif du traducteur et l'influence de la traduction sur la création littéraire. Acteurs de ce cheminement, des traducteurs et traductrices, dans un mouvement autoréflexif, montrent enfin, dans une dernière partie, que ce travail, pour solitaire qu'il soit, est aussi accompagné et que le processus est alors infléchi et modulé quand d'autres voix s'en mêlent. Un texte conclusif en forme de coda replace les questions abordées dans une perspective historique et comparée, soulignant à nouveau la place de la traduction comme création dans l'évolution des langues, des pratiques traductives et de la littérature.

Les trois articles regroupés dans la première partie, intitulée «Réflexions théoriques", représentent trois visions de la traduction et de la traductologie et, par conséquent, trois approches de la question de la créativité et de la problématique de la traduction littéraire comme création. C'est toutefois la créativité, et donc la possibilité même, selon un cheminement individuel, d'aboutir à une production qui soit une création, que ces trois contributions examinent dans des contextes différents: traductologique pour Lance Hewson, littéraire et linguistique pour Chiara Denti, traductologique et idéologique pour Jean-René Ladmiral.

Dès l'introduction de son article, Hewson («Les paradoxes de la créativité en traduction littéraire») rappelle que «traduction ne rime pas avec créativité» et que «la traduction littéraire a ses propres enjeux». Il souligne ensuite les paradoxes de la créativité puisque celle-ci est invisible, impensable même pour le lecteur de la traduction. En dépit de cela, il revient au traductologue expert d'en analyser les ressorts afin de pouvoir l'évaluer. Selon Hewson, la créativité en traduction littéraire se distingue de la création littéraire car elle n'est pas un phénomène global, mais survient plutôt en différents endroits du texte. Elle n'est pas pour autant nécessairement liée à la présence de difficultés puisque même une langue simple peut s'avérer redoutable à traduire. L'auteur tente ainsi de cerner les caractéristiques de la traduction créative, ce qui l'amène à conclure que nombre d'approches du problème pèchent par «théorisation excessive» et qu'un nouveau cadrage s'avère nécessaire. Celui qu'il propose, à la faveur de cette contribution, "a pour vocation de permettre une évaluation du 
travail du traducteur dans l'optique de la créativité». Hewson insiste en particulier sur le rôle du traductologue, expert en capacité de dégager des pistes interprétatives et d'envisager des «variantes paraphrastiques» permettant de comparer la traduction offerte à d'autres possibilités que le traducteur lui-même a pu envisager avant de les écarter. Il insiste également sur la lecture du texte littéraire qui « requiert une double vision »: «micro-structurelle» et "macro-structurelle». Autrement dit, la créativité s'inscrit dans l'économie globale du texte à traduire: ne sera pas créative toute solution qui viendrait contrarier la cohérence du texte original. La créativité, selon Hewson, se caractérise, du côté du traducteur, par une "prise de risque» et du côté du traductologue et du lecteur, par la reconnaissance de «l'inattendu». Elle est «éphémère», «imprévisible» et «fragile».

Elle apparaît cependant moins «imprévisible» dans les cas où plusieurs langues cohabitent dans un texte, phénomène ancien - et lié aux langues vernaculaires - que la littérature postcoloniale et les travaux critiques sur cette littérature ont rendu plus familier. Le traducteur, habitué à des situations plus souvent monolingues, se trouve alors devant une hétérogénéité linguistique sans que pour autant le texte, dans sa globalité, présente d'incohérences, comme le montrent les exemples analysés par Denti dans son article «L'hétérolinguisme ou penser autrement la traduction ». Après avoir retracé l'histoire de l'hétérolinguisme, terme préféré à ceux de plurilinguisme et multilinguisme, elle explique comment ce phénomène peut permettre d'élaborer des modèles alternatifs de la traduction: «Puisque l'hétérolinguisme active un processus créatif, ces textes invalident l'argument selon lequel la traduction n'est qu'une pâle copie de l'original», nous dit-elle. Qui plus est, le statut même d'original s'estompe, le texte hétérolingue provenant lui-même de plusieurs sources. Or, trop souvent encore, cette hybridité linguistique est perçue comme un problème alors qu'au contraire elle est une «occasion de renouvellement de la pratique traduisante. Car, non seulement l'hétérolinguisme ouvre la possibilité de penser la traduction en dehors du modèle monolingue, mais il invite également à la considérer comme un acte créatif et innovant». Il est également innovant en ce qu'il suscite une pratique collective de la traduction permettant de pallier le monolinguisme fréquent du traducteur. L'hétérolinguisme ménage donc à la traduction un «espace de créativité» et c'est aux traducteurs qu'il appartient de recréer un univers langagier qui reflète celui du texte de départ.

Cette contribution, qui invite à penser autrement la traduction, pourrait mettre à mal les notions rebattues de «source» et «cible» sur lesquelles, dans l'article suivant, "Comment peut-on être sourcier? Critique du littéralisme en traduction», Ladmiral fonde sa critique du littéralisme, position traductologique contraire, selon lui, à la créativité. Le lecteur, au fil des pages, reconnaîtra non seulement la vigueur iconoclaste d'une expression qui se veut frappée au coin du bon sens, mais aussi les dichotomies et autres apories chères à l'auteur. Ladmiral se livre en effet à une critique corrosive, sinon sans appel, de la position des sourciers:

En réalité, la position des sourciers revient à l'illusion littéraliste qu'on pourrait faire l'économie de la créativité en traduction, et même spécifiquement en traduction littéraire. Il n'y a qu'à traduire! entend-on parfois. Voilà une idée reçue à laquelle cèdent souvent ceux qui ne connaissent rien à la traduction. C'est le degré zéro de la créativité a contrario, que je viens de mentionner comme le déni de la créativité en traduction. 
Cette illusion littéraliste qui conduit tout droit à cette «esthétique du littéralisme» qu'il fustige allègrement est, notamment, propre à Meschonnic et Berman, et ses versions ethnologisante et philologisante manifestent, selon Ladmiral, un «tournant idéologique», politique ou anthropologique, du discours sur la traduction. Ladmiral, s'appuyant sur ses travaux antérieurs, met ainsi en place dans cet article les fondements d'une évolution d'un discours visant à dénoncer l'impensé idéologique sousjacent aux «théories» de la traduction. Ce faisant, il entraîne son lecteur dans une critique non moins idéologique des positions des uns ou des autres, en particulier de celles des «sourciers». Il réaffirme avec force qu'un traducteur doit respecter sa langue, la langue cible, et qu'il lui faut se démarquer de la «haine de soi » tout comme de «l'idéalisation de l'autre». La possibilité de création est dans l'exercice même de son talent:

La traduction littéraire est bel et bien une création, mais sur le mode discret du talent. [...] Cette créativité traductive s'inscrit dans le cadre d'une esthétique classique, où la forme est serve. À cet égard, la traduction nous fournit un paradigme du classicisme, qui a pour idéal de ciseler une forme qui soit la mise en œuvre rigoureuse du fond: le texte cible de la traduction s'assigne aussi la mise en forme rigoureuse, c'est-à-dire "fidèle", de ce fond auquel pourvoit le texte source de l'original.

Assurément, seule une pratique cibliste de la traduction autorise la créativité.

Les auteurs de la deuxième partie, dont le titre «Les dessous de la créativité » est extrait de l'article de Ladmiral, explorent les manières dont une relation "concrète» entre traduction et création est mise en œuvre dans le cas de la traduction du poème d'une part, dans celui de l'influence de la traduction sur l'insertion d'une œuvre dans une culture d'accueil d'autre part, mais également lorsque la traduction est à l'origine de l'élaboration d'une théorie esthétique de l'écriture.

Dans son article, «(Im)possible coïncidence des textes: l'ordinaire de la création...", Maryvonne Boisseau établit, d'un point de vue théorique, une distinction entre les termes de créativité et création afin de problématiser la comparaison entre «traduction» et "création» («La traduction littéraire comme création»). En faisant observer la réversibilité des termes selon le point de vue à partir duquel on se place, elle montre que le rapport entre les deux n'est pas donné mais construit et que la nonconcordance des langues en est la condition. Elle note que si l'on peut tenter de repérer les traces d'un processus créatif dans le texte produit, il est sans doute vain d'en entreprendre la théorisation: "l'acte même de création [...] ne peut sourdre que du rapport singulier que le traducteur entretient avec sa propre langue». Aussi, le défi du traducteur est-il bien de révéler les potentialités de la langue d’accueil à partir de la réserve créative du texte à traduire. C'est au lecteur et au traductologue qu'il revient de faire la part de ce qui relève, dans la traduction, d' «automatismes» et de la singularité d'un style. Or, cette singularité ne peut être que le résultat d'un travail patient qui assume ses choix, effectués dans «la langue que l'on parle». C'est précisément ce que l'auteure appelle «l'ordinaire de la création", la trouvaille ou l'innovation n'en étant que la partie la plus visible. En effet, rester au plus près de la source peut aussi révéler une créativité à l'œuvre comme le montre la traduction des extraits 3 et 4 cités dans l'article où les écarts minimes pourraient laisser penser à une absence de créativité. Or, en l'occurrence, «[...] cette conformité à l'usage n'a rien à voir avec la banalité d'une traduction sans relief car elle répond au classicisme de l'anglais de Mahon et révèle l'ajustement possible de la langue française aux contraintes de l'anglais ». 
Spiros Macris («Un Baudelaire flamand: la traduction des Fleurs du Mal par Bert Decorte, (1946)») aborde, lui, la question de la créativité et de la création à la fois du point de vue formel et du point de vue plus large du «système» littéraire. Il montre comment le travail technique de traduction portant en particulier sur la métrique a été essentiel pour favoriser une sorte de transplantation au sein d'un «système» littéraire qui ne laissait guère d'espace à la littérature d'expression flamande: «Aborder la traduction des Fleurs du Mal sous l'angle de la création conduit ainsi à relier le travail sur la langue, propre à l'activité de tout poète, et la constitution des moyens linguistiques et littéraires du traducteur, qui vient enrichir les moyens d'expression de cette littérature», écrit Macris. Le processus de traduction intègre alors des priorités visant à "[déplacer] insensiblement les repères du texte». La particularité de la traduction de Decorte est mise en lumière par la comparaison avec d'autres traductions des Fleurs du Mal: "La comparaison montre que la force de la traduction de Decorte est de recréer un univers cohérent et personnel, puisé dans un particularisme culturel et linguistique en pleine affirmation et dont il exalte la force expressive.» L'étude de Macris, extrêmement précise et minutieuse, conforte l'idée que la traduction de la poésie relève d'une tekhnê, pour reprendre le terme grec utilisé par Cassin, mais également qu'elle est tributaire d'un contexte historique, littéraire, linguistique et culturel au sein duquel elle sert de truchement:

Les Fleurs du Mal donne à Decorte la possibilité d'explorer ses capacités créatrices. Le poète français fait de lui un poète davantage flamand. Ce paradoxe apparent tient aux rapports que la littérature en Flandre a tissés avec la littérature française, notamment par le truchement des mouvements d'avant-garde francophones bruxellois. Le traducteur est d'abord un lecteur formé par sa propre littérature. La traduction qu'il entreprend doit être placée dans le prolongement de cette lecture - et écriture - comme une expérience individuelle nécessairement déterminée, par opposition à une approche textuelle se proposant une manière de reconstruction. La liberté qui en résulte trouve notamment son expression dans les continuités que l'on découvre entre la traduction de Decorte et son œuvre, entre son œuvre et la situation de la littérature flamande.

Si la traduction des Fleurs du Mal de Baudelaire par Decorte a contribué à l'essor d'une littérature flamande, celle de Sesame and Lilies et The Bible of Amiens de Ruskin par Proust est à l'origine de la conception esthétique présidant à l'écriture de À la recherche du temps perdu. C'est l'hypothèse proposée par Younès Ez-Zouaine qui, dans sa contribution "Proust traducteur de Ruskin...», soutient l'idée d'un pillage de Ruskin par Proust au moyen de la traduction. Dans ce cas, la traduction est, pour ainsi dire, instrumentalisée au service de l'élaboration d'une sorte de théorie de l'esthétique mise en œuvre par l'écrivain lors de l'écriture d'À la recherche du temps perdu. Ni le processus de traduction ni la traduction elle-même ne sont analysés ici en termes de processus créatif ou de création, mais la traduction, aussi approximative qu'elle soit, permet à Proust - qui ne maîtrisait pas l'anglais - de s'approprier ce que l'auteur appelle des «esthétèmes» («unités discontinues d'un discours esthétique considéré comme un système»). Le processus est ainsi analysé comme un moment de «cristallisation » de ce que l'œuvre de Ruskin peut apporter à l'écrivain en termes d'élaboration théorique d'une esthétique scripturale tandis que la traduction équivaut à la «désintégration» du texte de Ruskin afin que soit rendu possible le transfert de ses "esthétèmes» vers un autre cadre théorique, celui de l'écrivain qui l'élabore parallèlement à la traduction: "S’apparentant à la critique littéraire et au pastiche 
qu'il pratique pendant toute la période précédant la rédaction de $A$ la recherche $d u$ temps perdu, la traduction lui permet de mûrir autrement sa conception de l'écriture et de l'art». Dans cet article, très documenté, l'auteur s'intéresse au traducteur, Proust en l'occurrence, qui cherche dans les œuvres de Ruskin qu'il s'efforce de comprendre par la traduction de quoi nourrir sa propre réflexion esthétique en s'appropriant les idées exprimées pour mieux les infléchir vers ses propres conceptions.

La troisième section, "Traduire sur le vif», s'éloigne de considérations théoriques pour privilégier la parole des traducteurs eux-mêmes. Professionnels de la traduction, Kenneth Berri, Agata Gołębiewska et Michel Bataillon rendent leur expérience accessible au lecteur. Leurs contributions, qui traitent respectivement d'écriture romanesque et théâtrale, mettent en relief la place de la collaboration dans le processus de création ainsi que sa grande valeur: loin d'être une contrainte, ou un frein, la collaboration facilite l'émergence d'une version plus riche. Comme l'écrit Gołębiewska, «c'est la création collective d'une œuvre qui rend à la traduction toute sa dimension créative, et non le processus solitaire de l'écriture».

En premier lieu, Berri, traducteur d'un roman de Maïca Sanconie (De troublants détours), s'interroge sur le statut phénoménologique de la traduction, «fruit d'une poïesis, d'un acte de création» et situe d'emblée la situation de collaboration entre auteur et traducteur sur le plan de la créativité et de l'interprétation. Puis, comme le titre l'annonce («Pont de suspension. La collaboration auteur-traducteur mise à nu»), l'auteur développe la métaphore du pont qui représente à la fois la distance entre la source et la cible et le lieu d'une suspension au cours de laquelle traducteur et auteur échangent questions et commentaires. Berri insiste sur le travail du traducteur qui «ne cesse de faire et de défaire», mettant en lumière à la fois le façonnage d'un matériau et la part de l'imagination dans la représentation visuelle du texte. L'«alliance» traducteur-auteur crée un nouvel espace de convergence et de cohabitation entre les textes si bien que la dernière version produite, fruit d'une négociation et d'un amalgame des différents états discutés, est une traduction enrichie par l'interprétation partagée. Elle offre aussi «une strate de plus», méta-traductive, au palimpseste du texte en devenir. La métaphore du «pont sinueux» est renforcée par l'idée des "points de suspension» comme autant d'étapes dans le dialogue auteur-traducteur, expérience paradoxale de transparence et d'opacité, de non-finitude de l'écriture tout comme de son approximation. Cette conversation, qui n'est pas sans rappeler le Dialogue sur la traduction de la poétesse canadienne Anne Hébert et de son traducteur, Frank Scott, lors de la traduction du poème Tombeau des rois, est véritablement la confrontation d'une interprétation d'un côté et d'une intention (dirait-on «vouloirdire»?) de l'autre. Elle révèle non pas une hiérarchie mais une collaboration fraternelle, d'égal à égale, et une réciprocité qui fait du texte final un reflet prismatique de l'original. L'expérience elle-même répond, de fait, à l'opposition simpliste de «source» et «cible» en montrant qu'il existe un tiers espace entre les deux, lieu même de la création.

Ce tiers espace peut cependant tout envahir lorsqu'il s'agit de traduire sur scène. Dans l'article suivant, "Le traducteur dans un théâtre à mille temps», Gołębiewska examine l'évolution du théâtre et ses conséquences sur la traduction : en effet, la mise en scène est aujourd'hui considérée comme une écriture scénique dont la logique est indépendante de celle du texte écrit, ce qui a pour conséquence une reconfiguration de la scène actuelle. On sort du «schéma présentant une œuvre figée» pour faire la 
part belle, dans certains cas, à l'improvisation, et surtout à la création collective où chacun, y compris le traducteur, est acteur du projet théâtral. Une scène élargie englobe aussi les spectateurs qui participent ainsi à la création. Dans ce contexte, le texte évolue au fil des répétitions et le traducteur, qui ne peut s'appuyer sur un texte fixé d'avance, doit travailler en étroite collaboration avec le dramaturge et le metteur en scène. À cet égard, nous dit Gołębiewska,

la situation de la traduction dans le spectacle ou quasiment sur scène se rapproche de la situation pragmatique de l'interprète; et les risques, pour le traducteur comme pour la traduction, sont sans doute plus grands que dans une situation ordinaire. Et pourtant, on ne parlera sans doute pas d'«interprétation» au sens où on l'entend quand il s'agit d'interprétation simultanée ou consécutive. La raison en est, bien entendu, le jeu scénique et l'enjeu de la théâtralité ainsi que la présence des spectateurs.

Il arrive alors que se pose la question du transfert du sens et de la nécessité de la traduction, y compris dans un contexte international. La compréhension du texte joué va reposer essentiellement sur le jeu des acteurs et «leur force d'interprétation ", le «traducteur-interprète», présent sur le plateau, allant jusqu'à se transformer en comédien. Dans une seconde partie, l'auteur aborde la question des sur-titres dont elle se demande s'il ne s'agit pas de "l'ultime manifestation de la traduction", question à laquelle l'article suivant, «Faust I \& II. Chronique d'un sur-titrage», répondra.

Le sur-titrage est en effet un cas particulier de traduction nécessitant des aptitudes multiples, notamment d'interprète et de rédacteur. C'est une traduction qui, selon Bataillon, diffère de la traduction pour la scène et de la traduction littéraire tout en étant dérivée des deux. Elle suppose une maîtrise de contraintes techniques particulières que l'on retrouve dans la traduction audiovisuelle: le sur-titre doit être calibré, cohérent et lisible; il suppose le découpage préalable du texte joué et comporte un nombre de signes fixé par la capacité d'embrasser d'un regard la totalité du surtitre. En évoquant l'histoire récente du sur-titrage (le sur-titrage est, selon l'auteur, né à Avignon en 1996) et en relatant les étapes du sur-titrage de Faust I \& II à partir d'une adaptation scénique établie par Jutta Ferbers pour le metteur en scène Robert Wilson et le musicien Herbert Grönemeyer, et créé en 2015 à Avignon, Bataillon permet à son lecteur de pénétrer dans l'arrière-boutique du sur-titrage et de mesurer à quel point l'entreprise est délicate: «Jusqu'à la dernière représentation, les régisseurs des titres, les topeurs, modifient des détails, anodins ou très importants, car un surtitrage se gagne titre par titre». Dans le cas de Faust en particulier, le sur-titreur ne peut s'appuyer sur les traductions existantes. De plus, «l'objet scénique» est désormais cette œuvre fragmentaire et complexe de Wilson, Grönemeyer et Ferbers, maîtres d'œuvre du spectacle, collectivement, du fait de leurs rôles respectifs de metteur en scène, musicien ou traductrice; c'est, et ce n'est plus, le Faust I \& II de Goethe. Quant au rédacteur des sur-titres, «[il] ne peut s'en tenir à la lettre du texte, il doit sur-titrer à la fois les mots, les situations et les images», tenant la gageure de «construire» les titres «carton après carton" pour qu'ils disent à la fois «les mots et l'action». Quel est alors le statut du sur-titrage au regard de la traduction? C'est, finalement, une création éphémère, dans le vif du spectacle; Bataillon, fort de son expérience, en propose la définition suivante:

De même qu'il existe, dans la tradition des belles-lettres, des éditions juxtalinéaires des textes antiques, grecs et latins, ou des poètes contemporains, on peut légitimement 
considérer un sur-titrage comme une édition juxtalinéaire éphémère dans le temps et l'espace de la représentation.

Et, comme toujours, le temps accordé aux traducteurs-sur-titreurs est le moyen de parvenir à un résultat qui, pour être éphémère, n’en est pas moins efficace et beau.

Après avoir suivi les acteurs de la traduction dans leur atelier, le lecteur de ce volume pourra ensuite mettre en perspective les divers aspects, parfois contradictoires, des études proposées en retrouvant le fil de la problématique initiale, la traduction comme création, avec la contribution de Jean-Yves Masson. Historien de la traduction, comparatiste, l'auteur rappelle quelques vérités de La Palisse qui, pour évidentes qu'elles soient, n'en sont pas moins sources de réflexion. Un texte traduit, par exemple, «a en réalité deux auteurs » bien qu'aucun des mots de la traduction ne soit de l'auteur de l'ouvrage traduit. Autre constat: différentes traductions proposent différentes versions d'une même œuvre. Le lecteur ordinaire, normal, ne peut donc que «faire confiance» au traducteur. Pour autant, comme le souligne Masson, ce lecteur est soupçonneux et prompt à accuser le traducteur de maladresse, voire d'incompétence. Aussi, pour échapper à l'accusation de traîtrise, le traducteur, la traductrice, peut être tenté(e) de «lisser» les aspérités du texte, non pas tant en vertu d'une tendance ethnologisante que pour se protéger, observe-t-il. Cette situation est bien connue et n'y échappent guère que les traducteurs dont la traduction est passée à la postérité: Masson cite les exemples de Jacques Amyot, Baudelaire, et Pope dont les traductions de Plutarque, Poe et Homère, en dépit de leurs imperfections, «font œuvre», appartenant désormais pleinement à la littérature qui les a accueillies. La réalité est de fait nuancée et la considération accordée à la traduction par l'histoire de la littérature varie selon les pays, de la traduction perçue comme texte apocryphe à la traduction intégrée pleinement à l'œuvre d'un auteur. Aussi Masson appelle-t-il de ses vœux la reconnaissance que «la traduction est une forme de création» dont le régime propre est «d'être une réécriture sous contrainte». Néanmoins, parce qu’on ne crée pas ex nihilo, cette situation n'empêche ni la créativité ni la retraduction qui permet aux textes de «(re)naître» dans différentes langues, poursuivant la création initiale. La traduction est donc créatrice par son apport aux langues, en particulier dans le domaine de la lexicographie et, de façon générale, à la littérature, plus exactement au «patrimoine intellectuel», incluant non seulement la littérature mais les sciences et les techniques, l'histoire et la philosophie.

La problématique de la création semble donc secouer dans ses fondements la représentation traditionnelle de la traduction littéraire - c'est le thème de ce volume -, mais aussi philosophique, pragmatique et spécialisée. Le déni de création dont la traduction est l'objet, la difficulté d'accès au processus créatif lui-même, celle enfin de reconnaître et cerner la part de création dans le texte traduit, à la fois évidente et invisible, la mesure incertaine de la subjectivité du traducteur ou de la traductrice, sont autant de facteurs contribuant à une représentation faussée de la traduction et à une reconnaissance encore insuffisante de son rôle dans l'histoire de la littérature. Toutefois, comme le montrent ces articles, le partage d'une réflexion traductologique, théorique et empirique, et d'une expérience d'écriture et de traduction collective par les traducteurs eux-mêmes permet de mettre au jour les évidences refoulées. Les oppositions binaires s'effritent, les modèles se complexifient, les théories s'entrecroisent, les pratiques se modifient. Gageons donc, à l'instar de Masson, que ce qui est dû aux traducteurs et traductrices sera enfin pleinement reconnu. 


\section{NOTE}

1. La loi française ( $n^{\circ}$ 57-298 du 11 mars 1957 sur la propriété littéraire et artistique, article L112-3 du Code de la propriété intellectuelle) reconnaît aux traducteurs la dignité d'auteurs à part entière et les protège. Voir: www.legifrance.gouv.fr/affichCodeArticle.do;jsessionid=AA1067C6D10819A 051BC51803946EF19.tpdila09v_2?cidTexte=LEGITEXT000006069414\&idArticle=LEGIARTI000 006278877\&dateTexte $=20170808 \&$ categorieLien $=i d \#$ LEGIARTI000006278877, consulté le 8 août 2017. En dépit de cela, la reconnaissance publique de leur statut est loin d'être acquise et leur rôle dans la mécanique de l'édition méconnu, minimisé, sinon ignoré.

\section{RÉFÉRENCES}

CAssin, Barbara (2014): Il faut au moins deux langues pour savoir qu'on en parle une, Entretien avec Fabienne Durand-Bogaert. Genesis (Manuscrits-Recherche-Invention). 38(Traduire): 129-137.

CAssin, Barbara (2016): La traduction comme œuvre d'art. In: Barbara CAssin, dir. Après Babel, traduire. Marseille/Arles: Mucem/Actes Sud, 243-250.

Durand-Bogaert, Fabienne (2014): Les deux corps du texte. Genesis (Manuscrits-RechercheInvention). 38(Traduire):11-33.

Foenr-Janssens, Yasmina (2016): Les voyages des Mille et Une Nuits ou quand la traduction fabrique l'œuvre originale. In: Barbara CAssın, dir. Après Babel, traduire. Marseille/Arles: Mucem/Actes Sud, 121-129.

Lebrave, Jean-Louis (2014): Genèse d'une traduction. Comment Elmar Tophoven a annoté La Jalousie d'Alain Robbe-Grillet. Genesis (Manuscrits-Recherche-Invention). 38(Traduire): 35-68.

Prete, Antonio (2011/2013) : À l'ombre de l'autre langue. Pour un art de la traduction. (Traduit par Danièle Roвert) Cadenet: les éditions chemin de ronde.

Quillier, Patrick (2002): Sur l'oreille du traducteur. In: Daniel Delas, dir. Traduire 2. Paris: Les Belles Lettres, 161-175. 